\title{
Menger-Hausdorff metric and common fixed point theorems in Menger probabilistic $G$-metric spaces
}

\author{
Qiang Tu, Chuanxi Zhu* and Zhaogi Wu
}

"Correspondence:

chuanxizhu@126.com

Department of Mathematics,

Nanchang University, Nanchang,

330031, P.R. China

\section{Springer}

\begin{abstract}
In this paper, we introduce the concepts of generalized probabilistically bounded set $\Omega^{*}$ and Menger-Hausdorff metric $\widetilde{G}^{*}$ in Menger probabilistic G-metric spaces, and prove that $\left(\Omega^{*}, \widetilde{G}^{*}, \Delta\right)$ is also a Menger probabilistic $G$-metric space. Utilizing these concepts, we establish some common fixed point theorems for three hybrid pairs of mappings satisfying the common property (E.A) in Menger probabilistic G-metric spaces. Finally, an example is given to exemplify the theorems.
\end{abstract}

MSC: Primary 47H10; secondary $46 \mathrm{~S} 10$

Keywords: Menger-Hausdorff metric; Menger probabilistic G-metric space; common fixed point; common property (E.A)

\section{Introduction and preliminaries}

As a generalization of a metric space, the concept of a probabilistic metric space has been introduced by Menger [1, 2]. Fixed point theory in a probabilistic metric space is an important branch of probabilistic analysis, and many results on the existence of fixed points or solutions of nonlinear equations in Menger $P M$-spaces have been studied by many scholars (see e.g. [3, 4]). Egbert [5] defined the notion of the distance between two sets in a Menger $P M$-space, i.e., the so-called Menger-Hausdorff metric. In 2006, Mustafa and Sims [6] introduced the concept of a generalized metric space, and many fixed point results have been obtained by many authors (see e.g. [7-12]). On the other hand, Kaewcharoen and Kaewkhao [13] introduced the concept of a Hausdorff G-distance in a G-metric space. Moreover, Zhou et al. [14] defined the notion of a generalized probabilistic metric space or a $P G M$-space as a generalization of a $P M$-space and a $G$-metric space. After that, Zhu et al. [15] obtained some fixed point theorems in generalized probabilistic metric spaces. However, the concept of a Menger-Hausdorff $G^{*}$-metric in a $P G M$-space has not been introduced and studied yet.

To fill this gap, we introduce the concept of a generalized probabilistically bounded set and a Menger-Hausdorff $G^{*}$-metric in Menger probabilistic $G$-metric spaces, and we prove that $\left(\Omega^{*}, \widetilde{G}^{*}, \Delta\right)$ is also a Menger probabilistic $G$-metric space. Based on these, we obtain some useful results. As an application, we establish some common fixed point theorems for three hybrid pairs of mappings satisfying the common property $(E . A)$ in Menger probabilistic $G$-metric spaces. Finally, an example is given to illustrate the theorems.

(c) 2015 Tu et al. This article is distributed under the terms of the Creative Commons Attribution 4.0 International License (http://creativecommons.org/licenses/by/4.0/), which permits unrestricted use, distribution, and reproduction in any medium, provided you give appropriate credit to the original author(s) and the source, provide a link to the Creative Commons license, and indicate if changes were made. 
Throughout this paper, let $\mathbb{R}=(-\infty,+\infty), \mathbb{R}^{+}=[0,+\infty)$, and $\mathbb{Z}^{+}$be the set of all positive integers.

A mapping $F: \mathbb{R} \rightarrow \mathbb{R}^{+}$is called a distribution function if it is nondecreasing leftcontinuous with $\sup _{t \in \mathbb{R}} F(t)=1$ and $\inf _{t \in \mathbb{R}} F(t)=0$.

We shall denote by $\mathscr{D}$ the set of all distribution functions while $H$ will always denote the specific distribution function defined by

$$
H(t)= \begin{cases}0, & t \leq 0 \\ 1, & t>0\end{cases}
$$

A mapping $\Delta:[0,1] \times[0,1] \rightarrow[0,1]$ is called a triangular norm (for short, a $t$-norm) if the following conditions are satisfied:

(1) $\Delta(a, 1)=a$;

(2) $\Delta(a, b)=\Delta(b, a)$;

(3) $a \geq b, c \geq d \Rightarrow \Delta(a, c) \geq \Delta(b, d)$;

(4) $\Delta(a, \Delta(b, c))=\Delta(\Delta(a, b), c)$.

A typical example of $t$-norm is $\Delta_{m}$, where $\Delta_{m}(a, b)=\min \{a, b\}$, for each $a, b \in[0,1]$.

Remark 1.1 From (4), it is not difficult to find that

$$
\begin{aligned}
\Delta(\Delta(a, b), \Delta(c, d)) & =\Delta(\Delta(\Delta(a, b), c), d)=\Delta(\Delta(\Delta(a, c), b), d) \\
& =\Delta(\Delta(a, c), \Delta(b, d))=\cdots .
\end{aligned}
$$

Definition 1.1 [16] A triplet $(X, \mathscr{F}, \Delta)$ is called a Menger probabilistic metric space (for short, a Menger $P M$-space) if $X$ is a nonempty set, $\Delta$ is a $t$-norm and $\mathscr{F}$ is a mapping from $X \times X$ into $\mathscr{D}$ satisfying the following conditions (we denote $\mathscr{F}(x, y)$ by $F_{x, y}$ ):

(MS-1) $F_{x, y}(t)=H(t)$ for all $t \in R$ if and only if $x=y$;

(MS-2) $F_{x, y}(t)=F_{y, x}(t)$ for all $t \in R$;

(MS-3) $F_{x, y}(t+s) \geq \Delta\left(F_{x, z}(t), F_{z, y}(s)\right)$ for all $x, y, z \in X$ and $t, s \geq 0$.

Let $(X, \mathscr{F}, \Delta)$ be a $P M$-space and $A$ be a nonempty subset of $X$. Then the function

$$
D_{A}(t)=\sup _{s<t} \inf _{x, y \in A} F_{x, y}(s), \quad t \in \mathbb{R}
$$

is called the probabilistic diameter of $A$. If $\sup _{t>0} D_{A}(t)=1$, then $A$ is said to be probabilistically bounded.

Let $(X, \mathscr{F}, \Delta)$ be a Menger $P M$-space and $\Omega$ be the family of all nonempty probabilistically bounded $\mathscr{T}$-closed subsets of $X$. For any $A, B \in \Omega$, define the distribution functions as follows:

$$
\begin{aligned}
& \tilde{\mathscr{F}}(A, B)(t)=\tilde{F}_{A, B}(t)=\sup _{s<t} \Delta\left(\inf _{x \in A} \sup _{y \in B} F_{x, y}(s), \inf _{y \in B} \sup _{x \in A} F_{x, y}(s)\right), \quad s, t \in \mathbb{R}, \\
& \mathscr{F}(x, A)(t)=F_{x, A}(t)=\sup _{s<t} \sup _{y \in A} F_{x, y}(s), \quad s, t \in \mathbb{R},
\end{aligned}
$$

where $\tilde{\mathscr{F}}$ is called the Menger-Hausdorff metric induced by $\mathscr{F}$. 
Lemma 1.1 [16] Let $(X, \mathscr{F}, \Delta)$ be a Menger PM-space. Then for any $A, B, C \in \Omega$ and any $x, y \in X$, we have the following:

(i) $\tilde{F}_{A, B}(t)=1$ if and only if $A=B$;

(ii) $F_{x, A}(t)=1$ if and only if $x \in A$;

(iii) for any $x \in A, F_{x, B}(t) \geq \tilde{F}_{A, B}(t)$, for all $t \geq 0$;

(iv) $F_{x, A}\left(t_{1}+t_{2}\right) \geq \Delta\left(F_{x, y}\left(t_{1}\right), F_{y, A}\left(t_{2}\right)\right)$, for all $t_{1}, t_{2} \geq 0$;

(v) $F_{x, A}\left(t_{1}+t_{2}\right) \geq \Delta\left(F_{x, B}\left(t_{1}\right), F_{A, B}\left(t_{2}\right)\right)$, for all $t_{1}, t_{2} \geq 0$;

(vi) $\tilde{F}_{A, C}\left(t_{1}+t_{2}\right) \geq \Delta\left(\tilde{F}_{A, B}\left(t_{1}\right), \tilde{F}_{B, C}\left(t_{2}\right)\right)$, for all $t_{1}, t_{2} \geq 0$.

Definition 1.2 [14] A Menger probabilistic G-metric space (for brevity, a $P G M$-space) is a triple $\left(X, G^{*}, \Delta\right)$, where $X$ is a nonempty set, $\Delta$ is a continuous $t$-norm and $G^{*}$ is a mapping from $X \times X \times X$ into $\mathscr{D}\left(G_{x, y, z}^{*}\right.$ denote the value of $G^{*}$ at the point $\left.(x, y, z)\right)$ satisfying the following conditions:

(PGM-1) $G_{x, y, z}^{*}(t)=1$ for all $x, y, z \in X$ and $t>0$ if and only if $x=y=z$;

(PGM-2) $G_{x, x, y}^{*}(t) \geq G_{x, y, z}^{*}(t)$ for all $x, y, z \in X$ with $z \neq y$ and $t>0$;

(PGM-3) $G_{x, y, z}^{*}(t)=G_{x, z y}^{*}(t)=G_{y, x, z}^{*}(t)=\cdots$ (symmetry in all three variables);

(PGM-4) $G_{x, y, z}^{*}(t+s) \geq \Delta\left(G_{x, a, a}^{*}(s), G_{a, y, z}^{*}(t)\right)$ for all $x, y, z, a \in X$ and $s, t \geq 0$.

Definition 1.3 [14] Let $\left(X, G^{*}, \Delta\right)$ be a Menger $P G M$-space and $x_{0}$ be any point in $X$. For any $\epsilon>0$ and $\delta$ with $0<\delta<1$, and $(\epsilon, \delta)$-neighborhood of $x_{0}$ is the set of all points $y$ in $X$ for which $G_{x_{0}, y, y}^{*}(\epsilon)>1-\delta$ and $G_{y, x_{0}, x_{0}}^{*}(\epsilon)>1-\delta$. We write

$$
N_{x_{0}}(\epsilon, \delta)=\left\{y \in X: G_{x_{0}, y, y}^{*}(\epsilon)>1-\delta, G_{y, x_{0}, x_{0}}^{*}(\epsilon)>1-\delta\right\},
$$

which means that $N_{x_{0}}(\epsilon, \delta)$ is the set of all points $y$ in $X$ for which the probability of the distance from $x_{0}$ to $y$ being less than $\epsilon$ is greater than $1-\delta$.

Lemma 1.2 [14] Let $\left(X, G^{*}, \Delta\right)$ be a Menger PGM-space. Then $\left(X, G^{*}, \Delta\right)$ is a Hausdorff space in the topology introduced by the family $\left\{N_{x_{0}}(\epsilon, \delta)\right\}$ of $(\epsilon, \delta)$-neighborhoods.

Definition 1.4 [14] Let $\left(X, G^{*}, \Delta\right)$ be a $P G M$-space, and $\left\{x_{n}\right\}$ is a sequence in $X$.

(1) $\left\{x_{n}\right\}$ is said to be convergent to a point $x \in X$ (write $x_{n} \rightarrow x$ ), if for any $\epsilon>0$ and $0<\delta<1$, there exists a positive integer $M_{\epsilon, \delta}$ such that $x_{n} \in N_{x_{0}}(\epsilon, \delta)$ whenever $n>M_{\epsilon, \delta}$

(2) $\left\{x_{n}\right\}$ is called a Cauchy sequence, if for any $\epsilon>0$ and $0<\delta<1$, there exists a positive integer $M_{\epsilon, \delta}$ such that $G_{x_{n}, x_{m}, x_{l}}^{*}(\epsilon)>1-\delta$ whenever $n, m, l>M_{\epsilon, \delta}$

(3) $\left(X, G^{*}, \Delta\right)$ is said to be complete, if every Cauchy sequence in $X$ converges to a point in $X$.

We can analogously prove the following lemma as in Menger $P M$-spaces.

Lemma 1.3 Let $\left(X, G^{*}, \Delta\right)$ be a Menger PGM-space with $\Delta$ a continuoust-norm, $\left\{x_{n}\right\},\left\{y_{n}\right\}$, and $\left\{z_{n}\right\}$ be sequences in $X$ and $x, y, z \in X$, if $\left\{x_{n}\right\} \rightarrow x,\left\{y_{n}\right\} \rightarrow y$ and $\left\{z_{n}\right\} \rightarrow z$ as $n \rightarrow \infty$. Then

(1) $\liminf _{n \rightarrow \infty} G_{x_{n}, y_{n}, z_{n}}^{*}(t) \geq G_{x, y, z}^{*}(t)$ for all $t>0$;

(2) $G_{x, y, z}^{*}(t+0) \geq \lim \sup _{n \rightarrow \infty} G_{x_{n}, y_{n}, z_{n}}^{*}(t)$ for all $t>0$. 
Particularly, if $t_{0}$ is a continuous point of $G_{x, y, z}(\cdot)$, then $\lim _{n \rightarrow \infty} G_{x_{n}, y_{n}, z_{n}}\left(t_{0}\right)=G_{x, y, z}\left(t_{0}\right)$.

Definition 1.5 [17] A pair of self-mappings $S$ and $T$ on $X$ are said to be weakly compatible (or coincidentally commuting) if they commute at their coincidence point, i.e., if $\mathrm{Tu}=\mathrm{Su}$ for some $u \in X$ implies that $T S u=S T u$.

Definition 1.6 [18] Let $F_{1}, F_{2} \in \mathscr{D}$. The algebraic sum $F_{1} \oplus F_{2}$ of $F_{1}$ and $F_{2}$ is defined by

$$
\left(F_{1} \oplus F_{2}\right)(t)=\sup _{t_{1}+t_{2}=t} \min \left\{F_{1}\left(t_{1}\right), F_{2}\left(t_{2}\right)\right\}
$$

for all $t \in \mathbb{R}$.

As a generalization, we give the following definition.

Definition 1.7 Let $F_{1}, F_{2}, F_{3} \in \mathscr{D}$. The algebraic sum $F_{1} \oplus F_{2} \oplus F_{3}$ of $F_{1}, F_{2}$, and $F_{3}$ is defined by

$$
\left(F_{1} \oplus F_{2} \oplus F_{3}\right)(t)=\sup _{t_{1}+t_{2}+t_{3}=t} \min \left\{F_{1}\left(t_{1}\right), F_{2}\left(t_{2}\right), F_{3}\left(t_{3}\right)\right\}
$$

for all $t \in \mathbb{R}$.

Remark 1.2 Let $F_{3}(t)=H(t)$. Then Definition 1.6 and Definition 1.7 are equivalent.

For two functions $f$ and $g, f>g$ means that $f(t) \geq g(t)$ and there exists some $t_{0}$ such that $f\left(t_{0}\right)>g\left(t_{0}\right)$.

Definition 1.8 [19] Let $f$ and $g$ be self-mappings of a set $X$. If $w=f x=g x$ for some $x$ in $X$, then $x$ is called a coincidence point of $f$ and $g$, and $w$ is called point of coincidence of $f$ and $g$.

In the sequel, we will denote by $C(f, F)$ the set of all coincidence points of $f$ and $F$.

We recall the definitions of property $(E . A)$ for a hybrid pair of mappings and common property $(E . A)$ for two hybrid pairs of mappings in Menger PM-spaces.

Definition 1.9 [20] Let $(X, \mathscr{F}, \Delta)$ be a Menger $P M$-space, $(\Omega, \tilde{\mathscr{F}}, \Delta)$ be the induced Menger $P M$-space, $f: X \rightarrow X$ be a self-mapping and $F: X \rightarrow \Omega$ be a multivalued mapping. A pair of mappings $(f, F)$ is said to satisfy the property $(E . A)$, if there exist a sequence $\left\{x_{n}\right\}$ in $X$, some $a \in X$, and $A \in \Omega$, such that $\lim _{n \rightarrow \infty} f x_{n}=a \in A=\lim _{n \rightarrow \infty} F x_{n}$.

Definition 1.10 [20] Let $(X, \mathscr{F}, \Delta)$ be a Menger $P M$-space and $(\Omega, \tilde{\mathscr{F}}, \Delta)$ be the induced Menger $P M$-space, $f, g: X \rightarrow X$, and $F, G: X \rightarrow \Omega$. Two pairs of mappings $(f, F)$ and $(g, G)$ are said to satisfy the common property $(E . A)$ if there exist two sequences $\left\{x_{n}\right\},\left\{y_{n}\right\}$ in $X$, some $u \in X$ and $A, B \in \Omega$, such that

$$
\lim _{n \rightarrow \infty} F x_{n}=A, \quad \lim _{n \rightarrow \infty} G y_{n}=B, \quad \lim _{n \rightarrow \infty} f x_{n}=\lim _{n \rightarrow \infty} g y_{n}=u \in A \cap B .
$$




\section{Menger-Hausdorff metric in Menger PGM-spaces}

In this section, we first introduce some new concepts in Menger PGM-spaces, and then establish some useful results in Menger PGM-spaces.

Definition 2.1 Let $A$ be a nonempty subset of $X$. The function $D_{A}^{*}$ defined by

$$
D_{A}^{*}(t)=\sup _{s<t} \inf _{p, q, r \in A} G_{p, q, r}^{*}(s)
$$

is called the generalized probabilistic diameter of $A$.

Definition 2.2 A nonempty subset $A$ of $X$ is said to be

(1) generalized probabilistically bounded, if $\sup _{t>0} D_{A}^{*}(t)=1$;

(2) generalized probabilistically semi-bounded, if $0<\sup _{t>0} D_{A}^{*}(t)<1$;

(3) generalized probabilistically unbounded, if $\sup _{t>0} D_{A}^{*}(t)=0$.

Lemma 2.1 If $A$ and $B$ are two nonempty subsets of $X$, then

$$
D_{A \cup B}^{*}(x+y) \geq \Delta\left(D_{A}^{*}(x), D_{B}^{*}(y)\right) .
$$

Proof Let $x, y$ be given, for (2.1), we first prove that

$$
\inf _{p, q, r \in A \cup B} G_{p, q, r}^{*}(x+y) \geq \Delta\left(\inf _{p, q, r \in A} G_{p, q, r}^{*}(x), \inf _{p, q, r \in B} G_{p, q, r}^{*}(y)\right) .
$$

Case (1):

$$
\inf _{p, q, r \in A \cup B} G_{p, q, r}^{*}(x+y)=\inf _{\substack{p \in A \\ q, r \in B}} G_{p, q, r}^{*}(x+y) .
$$

For any $p, q, r \in X$, we have

$$
G_{p, q, r}^{*}(x+y) \geq \Delta\left(G_{p, a, a}^{*}(x), G_{a, q, r}^{*}(y)\right) .
$$

Taking the infimum on both sides of this inequality as $p$ ranges over $A$, $a$ ranges over $A \cap B$, and $r, q$ range over $B$, and using (2.3), we have

$$
\begin{aligned}
\inf _{p, q, r \in A \cup B} G_{p, q, r}^{*}(x+y) & =\inf _{\substack{p \in A \\
q, r \in B}} G_{p, q, r}^{*}(x+y) \geq \inf _{\substack{p \in A, q, r \in B \\
a \in A \cap B}} \Delta\left(G_{p, a, a}^{*}(x), G_{a, q, r}^{*}(y)\right) \\
& \geq \Delta\left(\inf _{\substack{p \in A \\
a \in A \cap B}} G_{p, a, a}^{*}(x), \inf _{\substack{q, r \in B \\
a \in A \cap B}} G_{a, q, r}^{*}(y)\right) \\
& \geq \Delta\left(\inf _{\substack{p \in A \\
a \in A}} G_{p, a, a}^{*}(x), \inf _{\substack{q, r \in B \\
a \in B}} G_{a, q, r}^{*}(y)\right) \\
& \geq \Delta\left(\inf _{p, q, r \in A} G_{p, q, r}^{*}(x), \inf _{p, q, r \in B} G_{p, q, r}^{*}(y)\right) .
\end{aligned}
$$

So, (2.2) is proved.

Case (2): $\inf _{p, q, r \in A \cup B} G_{p, q, r}^{*}(x+y)<\inf _{p \in A, B} G_{p, q, r}^{*}(x+y)$. Then one of the following equalities: 
(a) $\inf _{p, q, r \in A \cup B} G_{p, q, r}^{*}(x+y)=\inf _{p, q, r \in A} G_{p, q, r}^{*}(x+y)$,

(b) $\inf _{p, q, r \in A \cup B} G_{p, q, r}^{*}(x+y)=\inf _{p, q, r \in B} G_{p, q, r}^{*}(x+y)$

and

holds.

(c) $\inf _{p, q, r \in A \cup B} G_{p, q, r}^{*}(x+y)=\inf _{\substack{p, r \in A \\ q, q, r}} G_{p, r}^{*}(x+y)$

If (a) holds, we have

$$
\begin{aligned}
\inf _{p, q, r \in A \cup B} G_{p, q, r}^{*}(x+y) & =\inf _{p, q, r \in A} G_{p, q, r}^{*}(x+y) \geq \inf _{p, q, r \in A} G_{p, q, r}^{*}(x) \\
& \geq \Delta\left(\inf _{p, q, r \in A} G_{p, q, r}^{*}(x), 1\right) \geq \Delta\left(\inf _{p, q, r \in A} G_{p, q, r}^{*}(x), \inf _{p, q, r \in B} G_{p, q, r}^{*}(y)\right) .
\end{aligned}
$$

Then (2.2) is proved.

Similarly, we can prove that (2.2) is satisfied if (b) or (c) holds.

Finally, by (2.2) and the continuity of $\Delta$, we have

$$
\begin{aligned}
D_{A \cup B}^{*}(x+y) & =\sup _{s+t<x+y} \inf _{p, q, r \in A \cup B} G_{p, q, r}^{*}(s+t) \geq \sup _{\substack{s<x \\
t<y}} \inf _{p, q, r \in A \cup B} G_{p, q, r}^{*}(s+t) \\
& \geq \Delta\left(\sup _{s<x} \inf _{p, q, r \in A} G_{p, q, r}^{*}(s), \sup _{t<y} \inf _{p, q, r \in B} G_{p, q, r}^{*}(y)\right)=\Delta\left(D_{A}^{*}(x), D_{B}^{*}(y)\right) .
\end{aligned}
$$

This completes the proof.

Lemma 2.2 Let $\left(X, G^{*}, \Delta\right)$ be a Menger PGM-space with a continuous t-norm.

(1) If $A$ is a generalized probabilistically bounded set, then $D_{A}^{*}$ is a distribution function.

(2) If $A, B \subseteq X$ are two generalized probabilistically bounded sets, then $A \cup B$ is also a generalized probabilistically bounded set.

Proof (1) Since $A$ is a generalized probabilistically bounded set, by Definition 2.1, it is easy to see that $D_{A}^{*}(t)$ is nondecreasing in $t, D_{A}^{*}(0)=0, \sup _{t>0} D_{A}^{*}(t)=1$ and $D_{A}^{*}(t)$ is leftcontinuous in $t$. This shows that $D_{A}^{*}(t)$ is a distribution function.

(2) Since $A$ and $B$ are generalized probabilistically bounded sets, from Lemma 2.1 and the continuity of $\Delta$, we have $\sup _{t>0} D_{A \cup B}^{*}(t) \geq \Delta\left(\sup _{t>0} D_{A}^{*}\left(\frac{t}{2}\right), \sup _{t>0} D_{B}^{*}\left(\frac{t}{2}\right)\right)=\Delta(1,1)=1$. This completes the proof.

Remark 2.1 By Lemma 2.2(2), we claim that if $A, B, C$ are generalized probabilistically bounded sets, then $A \cup B \cup C$ is also a generalized probabilistically bounded set.

In the remainder of this paper, we always assume that $\left(X, G^{*}, \Delta\right)$ is a Menger $P G M$-space with a continuous $t$-norm $\Delta$ and $\Omega^{*}$ be the family of all nonempty $\mathscr{T}$-closed generalized probabilistically bounded sets.

Definition 2.3 For $A, B, C \in \Omega^{*}$, define the mapping $\widetilde{G}^{*}: \Omega^{*} \times \Omega^{*} \times \Omega^{*} \rightarrow \mathscr{D}$ by

$$
\widetilde{G}_{A, B, C}^{*}(t)=\min \left\{g_{A, B}(t), g_{B, C}(t), g_{C, A}(t)\right\},
$$

where $g_{A, B}(t)=\sup _{s<t} \Delta\left(\inf _{x \in A} \sup _{y \in B} g_{x, y}(s), \inf _{y \in B} \sup _{x \in A} g_{x, y}(s)\right), g_{x, y}(s)=\Delta\left(G_{x, x, y}^{*}(s)\right.$, $\left.G_{x, y, y}^{*}(s)\right)$.

Then $\widetilde{G}^{*}$ is called the Menger-Hausdorff metric induced by $G^{*}$. 
Definition 2.4 Let $A, B, C \in \Omega^{*}$ and $x, y, z \in X$.

(1) The generalized probabilistic distance between two points $x, y$ and a set $C$ is the function $\widetilde{G}_{x, y, C}^{*}(t)$ defined by

$$
\widetilde{G}_{x, y, C}^{*}(t)=\sup _{s<t} \sup _{z \in C} G_{x, y, z}^{*}(s), \quad t \geq 0 .
$$

(2) The generalized probabilistic distance between a point $x$ and two sets $B, C$ is the function $\widetilde{G}_{x, B, C}^{*}(t)$ defined by

$$
\widetilde{G}_{x, B, C}^{*}(t)=\min \left\{g_{x, B}(t), g_{B, C}(t), g_{x, C}(t)\right\}, \quad t \geq 0,
$$

where $g_{x, B}(t)=\sup _{s<t} \sup _{y \in B} g_{x, y}(s)$.

Lemma 2.3 For any $A, B, D \in \Omega^{*}$ and $a, b>0$, we have

$$
g_{A, B}(a+b) \geq \Delta\left(g_{A, D}(a), g_{D, B}(b)\right)
$$

Proof For any $x, y, z \in X$ and $s, t>0$, we have

$$
G_{x, y, y}^{*}(t+s) \geq \Delta\left(G_{x, z, z}^{*}(t), G_{z, y, y}^{*}(s)\right)
$$

and

$$
G_{x, x, y}^{*}(t+s) \geq \Delta\left(G_{x, x, z}^{*}(t), G_{z, z, y}^{*}(s)\right)
$$

for all $z \in D$. Using the continuity and monotonicity of $\Delta$, we have the following inequalities:

$$
\sup _{y \in B} G_{x, y, y}^{*}(t+s) \geq \Delta\left(\sup _{z \in D} G_{x, z, z}^{*}(t), \inf _{z \in D} \sup _{y \in B} G_{z, y, y}^{*}(s)\right)
$$

and

$$
\sup _{y \in B} G_{x, x, y}^{*}(t+s) \geq \Delta\left(\sup _{z \in D} G_{x, x, z}^{*}(t), \inf _{z \in D} \sup _{y \in B} G_{z, z, y}^{*}(s)\right) .
$$

Thus, we have

$$
\begin{aligned}
& \inf _{x \in A} \sup _{y \in B} G_{x, y, y}^{*}(t+s) \geq \Delta\left(\inf _{x \in A} \sup _{z \in D} G_{x, z, z}^{*}(t), \inf _{z \in D} \sup _{y \in B} G_{z, y, y}^{*}(s)\right), \\
& \inf _{x \in A} \sup _{y \in B} G_{x, x, y}^{*}(t+s) \geq \Delta\left(\inf _{x \in A} \sup _{z \in D} G_{x, x, z}^{*}(t), \inf _{z \in D} \sup _{y \in B} G_{z, z, y}^{*}(s)\right) .
\end{aligned}
$$

Similarly, we can get

$$
\begin{aligned}
& \inf _{y \in B} \sup _{x \in A} G_{x, x, y}^{*}(t+s) \geq \Delta\left(\inf _{z \in D} \sup _{x \in A} G_{x, x, z}^{*}(t), \inf _{y \in B} \sup _{z \in D} G_{z, z, y}^{*}(s)\right), \\
& \inf _{y \in B} \sup _{x \in A} G_{x, y, y}^{*}(t+s) \geq \Delta\left(\inf _{z \in D} \sup _{x \in A} G_{x, z, z}^{*}(t), \inf _{y \in B} \sup _{z \in D} G_{z, y, y}^{*}(s)\right) .
\end{aligned}
$$


Since $\Delta$ is associative, by combining (2.4), (2.5), (2.6), and (2.7), we obtain

$$
\begin{aligned}
& \inf _{x \in A} \sup _{y \in B} g_{x, y}(t+s) \\
& =\Delta\left(\inf _{x \in A} \sup _{y \in B} G_{x, y, y}^{*}(t+s), \inf _{x \in A} \sup _{y \in B} G_{x, x, y}^{*}(t+s)\right) \\
& \geq \Delta\left(\Delta\left(\inf _{x \in A} \sup _{z \in D} G_{x, z, z}^{*}(t), \inf _{z \in D} \sup _{y \in B} G_{z, y, y}^{*}(s)\right), \Delta\left(\inf _{x \in A} \sup _{z \in D} G_{x, x, z}^{*}(t), \inf _{z \in D} \sup _{y \in B} G_{z, z, y}^{*}(s)\right)\right) \\
& =\Delta\left(\Delta\left(\inf _{x \in A} \sup _{z \in D} G_{x, z, z}^{*}(t), \inf _{x \in A} \sup _{z \in D} G_{x, x, z}^{*}(t)\right), \Delta\left(\inf _{z \in D} \sup _{y \in B} G_{z, y, y}^{*}(s), \inf _{z \in D} \sup _{y \in B} G_{z, z, y}^{*}(s)\right)\right) \\
& =\Delta\left(\inf _{x \in A} \sup _{z \in D} g_{x, z}(t), \inf _{z \in D} \sup _{y \in B} g_{y, z}(s)\right) \\
& \inf _{y \in B} \sup _{x \in A} g_{x, y}(t+s) \\
& =\Delta\left(\inf _{y \in B} \sup _{x \in A} G_{x, y, y}^{*}(t+s), \inf _{y \in B} \sup _{x \in A} G_{x, x, y}^{*}(t+s)\right) \\
& \geq \Delta\left(\Delta\left(\inf _{z \in D} \sup _{x \in A} G_{x, z, z}^{*}(t), \inf _{y \in B} \sup _{z \in D} G_{z, y, y}^{*}(s)\right), \Delta\left(\inf _{z \in D} \sup _{x \in A} G_{x, x, z}^{*}(t), \inf _{y \in B} \sup _{z \in D} G_{z, z, y}^{*}(s)\right)\right) \\
& =\Delta\left(\Delta\left(\inf _{z \in D} \sup _{x \in A} G_{x, z, z}^{*}(t), \inf _{z \in D} \sup _{x \in A} G_{x, x, z}^{*}(t)\right), \Delta\left(\inf _{y \in B} \sup _{z \in D} G_{z, y, y}^{*}(s), \inf _{y \in B} \sup _{z \in D} G_{z, z, y}^{*}(s)\right)\right) \\
& =\Delta\left(\inf _{z \in D} \sup _{x \in A} g_{x, z}(t), \inf _{y \in B} \sup _{z \in D} g_{y, z}(s)\right) \text {. }
\end{aligned}
$$

By (2.8) and (2.9), we have

$$
\begin{aligned}
& g_{A, B}(a+b) \\
& \quad=\sup _{t+s<a+b} \Delta\left(\inf _{x \in A} \sup _{y \in B} g_{x, y}(t+s), \inf _{y \in B} \sup _{x \in A} g_{x, y}(t+s)\right) \\
& \geq \sup _{t+s<a+b} \Delta\left(\Delta\left(\inf _{x \in A} \sup _{z \in D} g_{x, z}(t), \inf _{z \in D} \sup _{y \in B} g_{y, z}(s)\right), \Delta\left(\inf _{z \in D} \sup _{x \in A} g_{x, z}(t), \inf _{y \in B} \sup _{z \in D} g_{y, z}(s)\right)\right) \\
& =\sup _{t+s<a+b} \Delta\left(\Delta\left(\inf _{x \in A} \sup _{z \in D} g_{x, z}(t), \inf _{z \in D} \sup _{x \in A} g_{x, z}(t)\right), \Delta\left(\inf _{z \in D} \sup _{y \in B} g_{y, z}(s), \inf _{y \in B} \sup _{z \in D} g_{y, z}(s)\right)\right) \\
& \geq \\
& \left.\geq \sup _{t<a} \Delta\left(\inf _{x \in A} \sup _{z \in D} g_{x, z}(t), \inf _{z \in D} \sup _{x \in A} g_{x, z}(t)\right), \sup _{s<b} \Delta\left(\inf _{z \in D} \sup _{y \in B} g_{y, z}(s), \inf _{y \in B} \sup _{z \in D} g_{y, z}(s)\right)\right) \\
& =\Delta\left(g_{A, D}(a), g_{D, B}(b)\right) .
\end{aligned}
$$

This completes the proof.

Theorem $2.1\left(\Omega^{*}, \widetilde{G}^{*}, \Delta\right)$ is a Menger PGM-space.

Proof First, we prove that $\widetilde{G}^{*}$ is a distribution function. By the definition of $\widetilde{G}^{*}(t)$, it is easy to see that $\widetilde{G}^{*}(t)$ is nondecreasing and left-continuous in $t$ and $\widetilde{G}^{*}(0)=0$. Now, we prove

$$
\sup _{t>0} \widetilde{G}^{*}(t)=1
$$

In fact, since $A, B, C \in \Omega^{*}$, we know $A \cup B \cup C \in \Omega^{*}$. By the continuity of $\Delta$, we have

$$
\sup _{t>0} \widetilde{G}^{*}(t)=\sup _{t>0} \min \left\{g_{A, B}(t), g_{B, C}(t), g_{C, A}(t)\right\}=\min \left\{\sup _{t>0} g_{A, B}(t), \sup _{t>0} g_{B, C}(t), \sup _{t>0} g_{C, A}(t)\right\}
$$


and

$$
\begin{aligned}
& \sup _{t>0} g_{A, B}(t)=\sup _{\substack{t>0 \\
s<t}} \Delta\left\{\Delta\left[\inf _{x \in A} \sup _{y \in B} G_{x, y, y}^{*}(s), \inf _{x \in A} \sup _{y \in B} G_{x, x, y}^{*}(s)\right],\right. \\
& \left.\Delta\left[\inf _{y \in B} \sup _{x \in A} G_{x, y, y}^{*}(s), \inf _{y \in B} \sup _{x \in A} G_{x, x, y}^{*}(s)\right]\right\} \\
& \geq \Delta\left\{\sup _{\substack{t>0 \\
s<t}} \Delta\left[\inf _{x \in A} \inf _{y \in B} G_{x, y, y}^{*}(s), \inf _{x \in A} \inf _{y \in B} G_{x, x, y}^{*}(s)\right],\right. \\
& \left.\sup _{\substack{t>0 \\
s<t}} \Delta\left[\inf _{y \in B} \inf _{x \in A} G_{x, y, y}^{*}(s), \inf _{y \in B} \inf _{x \in A} G_{x, x, y}^{*}(s)\right]\right\} \\
& \geq \Delta\left\{\Delta\left[\sup _{\substack{t>0 \\
s<t}} \inf _{x, y \in A \cup B} G_{x, y, y}^{*}(s), \sup _{\substack{t>0 \\
s<t}} \inf _{x, y \in A \cup B} G_{x, x, y}^{*}(s)\right],\right. \\
& \left.\Delta\left[\sup _{\substack{t>0 \\
s<t}} \inf _{x, y \in A \cup B} G_{x, y, y}^{*}(s), \sup _{\substack{t>0 \\
s<t}} \inf _{\substack{x \\
s<A \cup B}} G_{x, x, y}^{*}(s)\right]\right\}
\end{aligned}
$$

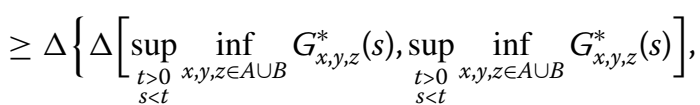

$$
\begin{aligned}
& \left.\Delta\left[\sup _{\substack{t>0 \\
s<t}} \inf _{x, y, z \in A \cup B} G_{x, y, z}^{*}(s), \sup _{\substack{t>0 \\
s<t}} \inf _{x, y, z \in A \cup B} G_{x, y, z}^{*}(s)\right]\right\} \\
& =\Delta\left\{\Delta\left[D_{A \cup B}^{*}, D_{A \cup B}^{*}\right], \Delta\left[D_{A \cup B}^{*}, D_{A \cup B}^{*}\right]\right\} \\
& =\Delta(\Delta(1,1), \Delta(1,1)) \\
& =1 \text {. }
\end{aligned}
$$

Similarly, we have $\sup _{t>0} g_{B, C}(t)=1$ and $\sup _{t>0} g_{C, A}(t)=1$. This shows that $\widetilde{G}^{*}$ is a mapping from $\Omega^{*} \times \Omega^{*} \times \Omega^{*}$ into $\mathscr{D}$.

Next, we will show that $\widetilde{G}^{*}(t)$ satisfies the following:

(1) $\widetilde{G}_{A, B, C}^{*}(t)=1$ for all $t>0$ if and only if $A=B$;

(2) $\widetilde{G}_{A, A, B}^{*}(t) \geq \widetilde{G}_{A, B, C}^{*}(t)$ for all $A, B, C \in \Omega^{*}$ with $B \neq C$ and $t>0$;

(3) $\widetilde{G}_{A, B, C}^{*}(t)=\widetilde{G}_{A, C, B}^{*}(t)=\widetilde{G}_{B, A, C}^{*}(t)=\cdots$ (symmetry in all three variables);

(4) $\widetilde{G}_{A, B, C}^{*}(t+s) \geq \Delta\left(\widetilde{G}_{A, D, D}^{*}(t), \widetilde{G}_{D, B, C}^{*}(t)\right)$ for all $A, B, C \in \Omega^{*}$ with $t>0$.

- (i) If $\widetilde{G}_{A, B, C}^{*}(t)=1$ for all $t>0$, then for any $\epsilon>0$, we have

$$
g_{A, B}(\epsilon)=g_{B, C}(\epsilon)=g_{C, A}(\epsilon)=1 .
$$

By $g_{A, B}(\epsilon)=1$, we have

$$
\begin{aligned}
& \sup _{s<\epsilon} \inf _{x \in A} \sup _{y \in B} \Delta\left(G_{x, x, y}^{*}(s), G_{x, y, y}^{*}(s)\right)=1, \\
& \sup _{s<\epsilon} \inf _{y \in B} \sup _{x \in A} \Delta\left(G_{x, x, y}^{*}(s), G_{x, y, y}^{*}(s)\right)=1 .
\end{aligned}
$$

From (2.10), it follows that $\sup _{s<\epsilon} \sup _{y \in B} \Delta\left(G_{x, x, y}^{*}(s), G_{x, y, y}^{*}(s)\right)=1$ for all $x \in A$. Therefore, for any $a \in A$ and $\lambda>0$, there exists $b^{*} \in B$, such that

$$
\Delta\left(G_{a, a, b^{*}}^{*}(\epsilon), G_{a, b^{*}, b^{*}}^{*}(\epsilon)\right)>1-\lambda
$$


So, we have

$$
G_{a, a, b^{*}}^{*}(\epsilon)>1-\lambda \quad \text { and } \quad G_{a, b^{*}, b^{*}}^{*}(\epsilon)>1-\lambda \text {. }
$$

This shows that the point $a$ is an accumulation point of $B$ and hence $a \in B$, i.e., $A \subseteq B$.

From (2.11), we can prove that $B \subseteq A$. Therefore, we have $A=B$.

Similarly, we can also prove that $B=C, C=A$. So, we have $A=B=C$.

Conversely, if $A=B=C$, then for any $t>0$, we have

$$
\widetilde{G}_{A, B, C}^{*}(t)=\min \left\{g_{A, A}(t), g_{A, A}(t), g_{A, A}(t)\right\}=g_{A, A}(t) .
$$

For any $s \in(0,1)$,

$$
\begin{aligned}
g_{A, A}(t) & \geq \Delta\left(\inf _{a \in A} \sup _{b \in A} g_{a, b}(s), \inf _{a \in A} \sup _{b \in A} g_{a, b}(s)\right) \\
& =\Delta\left(\inf _{a \in A} \sup _{b \in A} \Delta\left(G_{a, a, b}^{*}(s), G_{a, b, b}^{*}(s)\right), \inf _{a \in A} \sup _{b \in A} \Delta\left(G_{a, a, b}^{*}(s), G_{a, b, b}^{*}(s)\right)\right) \\
& =\Delta(1,1)=1 .
\end{aligned}
$$

Therefore (1) is satisfied.

- (ii) $\widetilde{G}_{A, A, B}^{*}(t)=\min \left\{g_{A, A}(t), g_{A, B}(t), g_{A, B}(t)\right\}=g_{A, B}(t) \geq \min \left\{g_{A, B}(t), g_{B, C}(t), g_{C, A}(t)\right\}=$ $\widetilde{G}_{A, B, C}^{*}(t)$. So, $(2)$ is satisfied.

- (iii) It is obvious that (3) holds.

- (iv) From Definition 2.3, we have

$$
\begin{aligned}
& \widetilde{G}_{A, B, C}^{*}(t+s)=\min \left\{g_{A, B}(t+s), g_{B, C}(t+s), g_{C, A}(t+s)\right\}, \\
& \widetilde{G}_{A, D, D}^{*}(t)=\min \left\{g_{A, D}(t), g_{D, D}(t), g_{A, D}(t)\right\}=g_{A, D}(t), \\
& \widetilde{G}_{D, B, C}^{*}(s)=\min \left\{g_{D, B}(s), g_{B, C}(s), g_{C, D}(s)\right\} .
\end{aligned}
$$

We just need to show

$$
\min \left\{g_{A, B}(t+s), g_{B, C}(t+s), g_{C, A}(t+s)\right\} \geq \Delta\left(g_{A, D}(t), \min \left\{g_{D, B}(s), g_{B, C}(s), g_{C, D}(s)\right\}\right) .
$$

In fact,

$$
\begin{aligned}
g_{B, C}(t+s) & \geq g_{B, C}(s) \geq \min \left\{g_{D, B}(s), g_{B, C}(s), g_{C, D}(s)\right\} \\
& \geq \Delta\left(g_{A, D}(t), \min \left\{g_{D, B}(s), g_{B, C}(s), g_{C, D}(s)\right\}\right), \\
g_{A, B}(t+s) & \geq \Delta\left(g_{A, D}(t), g_{D, B}(s)\right) \geq \Delta\left(g_{A, D}(t), \min \left\{g_{D, B}(s), g_{B, C}(s), g_{C, D}(s)\right\}\right), \\
g_{C, A}(t+s) & \geq \Delta\left(g_{A, D}(t), g_{D, C}(s)\right) \geq \Delta\left(g_{A, D}(t), \min \left\{g_{D, B}(s), g_{B, C}(s), g_{C, D}(s)\right\}\right) .
\end{aligned}
$$

So, (4) is also satisfied. This completes the proof.

Remark 2.2 By the proof process of Lemma 2.3 and Theorem 2.1, we can also prove that $(X, g, \Delta)$ and $\left(\Omega^{*}, g, \Delta\right)$ are Menger $P M$-spaces. We call $(X, g, \Delta)$ the $P M$-space induced by $\left(X, G^{*}, \Delta\right)$, and $\left(\Omega^{*}, g, \Delta\right)$ is the $P M$-space induced by $(X, g, \Delta)$. So, the properties in Lemma 1.1 can be applied to $(X, g, \Delta)$ and $\left(\Omega^{*}, g, \Delta\right)$. 
Example 2.1 Let $(X, d)$ be a metric space and $x, y, z \in X, G_{x, y, z}^{*}(t)=\frac{t}{t+\max \{d(x, y), d(y, z), d(x, z)\}}$ for all $t \geq 0$, then $\left(X, G^{*}, \Delta_{m}\right)$ is a Menger $P G M$-space. In fact, $G_{x, y, z}^{*}(0)=0, \sup _{t>0} G_{x, y, z}^{*}(t)=1$, and $G_{x, y, z}^{*}(t)$ is nondecreasing and continuous in $t$, so $G_{x, y, z}^{*}(t)$ is a distribution function. Obviously, $G_{x, y, z}^{*}(t)$ satisfy (PGM-1), (PGM-2), and (PGM-3). Next, we will show that (PGM-4) is also satisfied. Since $d(x, y) \leq d(x, a)+d(a, y)$ and $d(x, z) \leq d(x, a)+d(a, z)$, we have

$$
\begin{aligned}
G_{x, y, z}^{*}(t+s) & =\frac{t+s}{t+s+\max \{\max \{d(x, y), d(y, z), d(x, z)\}\}} \\
& \geq \frac{t+s}{t+s+\max \{d(x, a)+d(a, y), d(y, z), d(x, a)+d(a, z)\}} \\
& \geq \frac{t+s}{t+s+d(x, a)+\max \{d(a, y), d(y, z), d(a, z)\}} \\
& \geq \min \left\{\frac{t}{t+d(x, a)}, \frac{s}{s+\max \{d(a, y), d(y, z), d(a, z)\}}\right\} \\
& =\min \left\{G_{x, a, a}^{*}(t), G_{a, y, z}^{*}(s)\right\},
\end{aligned}
$$

which implies that (PGM-4) is satisfied. So $\left(X, G^{*}, \Delta_{m}\right)$ is a Menger $P G M$-space. Then

$$
g_{x, y}(t)=\min \left\{G_{x, x, y}^{*}(t), G_{x, y, y}^{*}(t)\right\}=\frac{t}{t+d(x, y)}
$$

and

$$
\begin{aligned}
g_{A, B}(t) & =\min \left\{\inf _{x \in A} \sup _{y \in B} \frac{t}{t+d(x, y)}, \inf _{y \in B} \sup _{x \in A} \frac{t}{t+d(x, y)}\right\} \\
& =\min \left\{\frac{t}{t+\inf _{x \in A} \sup _{y \in B} d(x, y)}, \frac{t}{t+\inf _{y \in B} \sup _{x \in A} d(x, y)}\right\} \\
& =\frac{t}{t+\max \left\{\inf _{x \in A} \sup _{y \in B} d(x, y), \inf _{y \in B} \sup _{x \in A} d(x, y)\right\}}=\frac{t}{t+\delta(A, B)} .
\end{aligned}
$$

Thus,

$$
\begin{aligned}
\widetilde{G}_{A, B, C}^{*}(t) & =\min \left\{g_{A, B}(t), g_{B, C}(t), g_{A, C}(t)\right\} \\
& =\min \left\{\frac{t}{t+\delta(A, B)}, \frac{t}{t+\delta(B, C)}, \frac{t}{t+\delta(A, C)}\right\} \\
& =\frac{t}{t+\max \{\delta(A, B), \delta(B, C), \delta(A, C)\}},
\end{aligned}
$$

where $\delta(A, B)=\max \left\{\inf _{x \in A} \sup _{y \in B} d(x, y), \inf _{y \in B} \sup _{x \in A} d(x, y)\right\}$. Then $\left(\Omega^{*}, \widetilde{G}_{A, B, C}^{*}(t), \Delta_{m}\right)$ is a Menger $P G M$-space induced by $\left(X, G^{*}, \Delta_{m}\right)$.

Theorem 2.2 Let $\left(\Omega^{*}, \widetilde{G}^{*}, \Delta\right)$ be a Menger PGM-space. Then for any $A, B, C, D \in \Omega^{*}$ and $x, y, z \in X$, we have the following:

(1) $\widetilde{G}_{x, B, C}^{*}(t)=1$ if and only if $x \in B=C$;

(2) $\widetilde{G}_{x, x, B}^{*}(t) \geq \widetilde{G}_{x, B, B}^{*}(t) \geq \widetilde{G}_{x, B, C}^{*}(t) \geq \widetilde{G}_{A, B, C}^{*}(t)$ for all $x \in A$ and $t \geq 0$;

(3) $\widetilde{G}_{x, B, C}^{*}(t+s) \geq \Delta\left(\widetilde{G}_{x, D, D}^{*}(t), \widetilde{G}_{D, B, C}^{*}(s)\right)$ for all $s, t \geq 0$;

(4) $\widetilde{G}_{x, y, C}^{*}(t+s) \geq \Delta\left(\widetilde{G}_{x, a, a}^{*}(t), \widetilde{G}_{a, y, C}^{*}(s)\right)$ for all $s, t \geq 0$ and $a \in X$. 
Proof (1) If $\widetilde{G}_{x, B, C}^{*}(t)=\min \left\{g_{x, B}(t), g_{B, C}(t), g_{x, C}(t)\right\}=1$, then we have $g_{x, B}(t)=g_{B, C}(t)=$ $g_{x, C}(t)=1$, which implies that $x \in B, x \in C, B=C$, that is, $x \in B=C$.

Conversely, it is obvious that $\widetilde{G}_{x, B, C}^{*}(t)=1$ holds.

(2) From Definition 2.4 and Lemma 1.1, we have

$$
\begin{aligned}
\widetilde{G}_{x, x, B}^{*}(t) & =\sup _{y \in B} G_{x, x, y}^{*}(t) \geq g_{x, B}(t)=\widetilde{G}_{x, B, B}^{*}(t) \geq \min \left\{g_{x, B}(t), g_{B, C}(t), g_{x, C}(t)\right\} \\
& =\widetilde{G}_{x, B, C}^{*}(t) \geq \min \left\{g_{A, B}(t), g_{B, C}(t), g_{A, C}(t)\right\}=\widetilde{G}_{A, B, C}^{*}(t) .
\end{aligned}
$$

So, (2) is proved.

(3) By Definition 2.4 and Lemma 1.1, we have $\widetilde{G}_{x, B, C}^{*}(t+s)=\min \left\{g_{x, B}(t+s), g_{B, C}(t+\right.$ $\left.s), g_{x, C}(t+s)\right\}$,

$$
\begin{aligned}
& g_{x, B}(t+s) \geq \Delta\left(g_{x, D}(t), g_{D, B}(s)\right)=\Delta\left(\widetilde{G}_{x, D, D}^{*}(t), g_{D, B}(s)\right) \geq \Delta\left(\widetilde{G}_{x, D, D}^{*}(t), \widetilde{G}_{D, B, C}^{*}(s)\right), \\
& g_{B, C}(t+s) \geq g_{B, D}(s) \geq \widetilde{G}_{D, B, C}^{*}(s) \geq \Delta\left(\widetilde{G}_{x, D, D}^{*}(t), \widetilde{G}_{D, B, C}^{*}(s)\right), \\
& g_{x, C}(t+s) \geq \Delta\left(g_{x, D}(t), g_{D, C}(s)\right)=\Delta\left(\widetilde{G}_{x, D, D}^{*}(t), g_{D, C}(s)\right) \geq \Delta\left(\widetilde{G}_{x, D, D}^{*}(t), \widetilde{G}_{D, B, C}^{*}(s)\right) .
\end{aligned}
$$

So, (3) is proved.

(4) By Lemma 1.1, we have

$$
\widetilde{G}_{x, y, C}^{*}(t+s)=\sup _{z \in C} G_{x, y, z}^{*}(t+s) \geq \sup _{z \in C} \Delta\left(\widetilde{G}_{x, a, a}^{*}(t), \widetilde{G}_{a, y, z}^{*}(s)\right)=\Delta\left(\widetilde{G}_{x, a, a}^{*}(t), \widetilde{G}_{a, y, C}^{*}(s)\right) .
$$

Remark 2.3 By (1), (2), and the proof of Lemma 2.2, it is easy to prove that $\widetilde{G}_{x, x, B}^{*}(t)=1$ if and only if $x \in B$, and $\widetilde{G}_{x, B, B}^{*}(t)=1$ if and only if $x \in B$.

\section{Common fixed point theorems in Menger PGM-spaces}

In this section, we will give some common fixed point theorems in Menger probabilistic $G$-metric spaces. To this end, we first introduce the concept of common property (E.A) for three hybrid pairs of mappings in Menger probabilistic G-metric spaces.

Definition 3.1 Let $\left(X, G^{*}, \Delta\right)$ be a Menger $P M$-space and $\left(\Omega^{*}, \widetilde{G}^{*}, \Delta\right)$ be the induced Menger $P M$-space, $f, h, r: X \rightarrow X$ and $F, H, R: X \rightarrow \Omega^{*}$. Three pairs of mappings $(f, F)$, $(h, H)$, and $(r, R)$ are said to satisfy the common property $(E . A)$ if there exist three sequences $\left\{x_{n}\right\},\left\{y_{n}\right\},\left\{z_{n}\right\}$ in $X$, some $u \in X$ and $A, B, C \in \Omega^{*}$, such that

$$
\begin{aligned}
& \lim _{n \rightarrow \infty} F x_{n}=A, \quad \lim _{n \rightarrow \infty} H y_{n}=B, \quad \lim _{n \rightarrow \infty} R y_{n}=C, \\
& \lim _{n \rightarrow \infty} f x_{n}=\lim _{n \rightarrow \infty} h y_{n}=\lim _{n \rightarrow \infty} r z_{n}=u \in A \cap B \cap C .
\end{aligned}
$$

We are now ready to give the common fixed point theorems in Menger probabilistic G-metric spaces.

Theorem 3.1 Let $\left(X, G^{*}, \Delta\right)$ be a Menger PGM-space with a continuous $t$-norm on $[0,1] \times$ $[0,1]$ and $\left(\Omega^{*}, \widetilde{G}^{*}, \Delta\right)$ be the induced Menger PGM-space. Suppose that $f, h, r: X \rightarrow X$ and $F, H, R: X \rightarrow \Omega^{*}$ are mappings satisfying the following conditions:

(1) $(f, F),(h, H)$, and $(r, R)$ satisfy the common property $(E . A)$; 
(2) $f(X), h(X)$, and $r(X)$ are $\mathscr{T}$-closed subsets of $X$;

(3) for any $x, y, z \in X$ with $F x, H y$, and $R z$ not all equal and some $1 \leq k \leq 3$,

$$
\widetilde{G}_{F x, H y, R z}^{*}>\min \left\{G_{f x, h y, r z}^{*}, \frac{3}{k}\left[\widetilde{G}_{F x, h y, r z}^{*} \oplus \widetilde{G}_{f x, H y, r z}^{*} \oplus \widetilde{G}_{f x, h y, R z}^{*}\right]\right\}
$$

where ${ }_{\frac{3}{k}}\left[\widetilde{G}_{F x, h y, r z}^{*} \oplus \widetilde{G}_{f x, H y, r z}^{*} \oplus \widetilde{G}_{f x, h y, R z}^{*}\right](t)$ means $\left[\widetilde{G}_{F x, h y, r z}^{*} \oplus \widetilde{G}_{f x, H y, r z}^{*} \oplus \widetilde{G}_{f x, h y, R z}^{*}\right]\left(\frac{3}{k} t\right)$.

Then $(f, F),(h, H)$, and $(r, R)$ each has a coincidence point. Moreover, ifffv $=f v$ for $v \in$ $C(f, F), h h v=h v$ for $v \in C(h, H)$, and $r r v=r v$ for $v \in C(r, R)$, then $f, h, r, F, H$, and $R$ have a common fixed point in $X$.

Proof Since $(f, F),(h, H)$, and $(r, R)$ satisfy the common property $(E . A)$, there exist $\left\{x_{n}\right\},\left\{y_{n}\right\},\left\{z_{n}\right\} \subset X$, some $u \in X$ and $A, B, C \in \Omega^{*}$, such that

$$
\begin{aligned}
& \lim _{n \rightarrow \infty} F x_{n}=A, \quad \lim _{n \rightarrow \infty} H y_{n}=B, \quad \lim _{n \rightarrow \infty} T z_{n}=C, \\
& \lim _{n \rightarrow \infty} f x_{n}=\lim _{n \rightarrow \infty} h y_{n}=\lim _{n \rightarrow \infty} r z_{n}=u \in A \cap B \cap C .
\end{aligned}
$$

Since $f(X)$ is $\mathscr{T}$-closed, there exists some $v \in X$, such that $u=f v$. We claim that $f v \in F v$. Suppose this is not true, then $f v \notin F v$. By $u=f v \in B$, we have $B \neq F v$. Thus, there exists some $t_{0}>0$, such that

$$
\widetilde{G}_{F v, B, C}^{*}\left(\frac{3 t_{0}}{k}\right)>\widetilde{G}_{F v, B, C}^{*}\left(t_{0}\right) \text {. }
$$

(Otherwise, for all $t>0, \widetilde{G}_{F v, B, C}^{*}(t)=\widetilde{G}_{F v, B, C}^{*}\left(\frac{3 t}{k}\right)=\cdots=\widetilde{G}_{F v, B, C}^{*}\left(\left(\frac{3}{k}\right)^{n} t\right) \rightarrow 1$ as $n \rightarrow \infty$, that is, $\widetilde{G}_{F v, B, C}^{*}(t)=1$, for all $t>0$, which is a contradiction.)

Without loss of generality, we can assume that $t_{0}$ is a continuous point of $\widetilde{G}_{F v, B, C}^{*}(\cdot)$. In fact, by the left continuity of the distribution function, we know that there exists some $\delta>0$, such that

$$
\widetilde{G}_{F v, B, C}^{*}\left(\frac{3 t}{k}\right)>\widetilde{G}_{F v, B, C}^{*}(t), \quad \forall t \in\left(t_{0}-\delta, t_{0}\right]
$$

Since the distribution function is nondecreasing, the discontinuous points are at most a countable set. Thus, when $t_{0}$ is not a continuous point of $\widetilde{G}_{F v, B, C}^{*}(\cdot)$, we can always choose a point $t_{1}$ in $\left(t_{0}-\delta, t_{0}\right]$ to replace $t_{0}$.

Noting that $\lim _{n \rightarrow \infty} f x_{n}=u \notin F v$ and $u \in B=\lim _{n \rightarrow \infty} H y_{n}$, we have $F v \neq \lim _{n \rightarrow \infty} H y_{n}$, so there exists some $n_{0} \in \mathbb{Z}^{+}$, such that for all $n \geq n_{0}, H y_{n} \neq F v$.

From (3.1) we know that

$$
\widetilde{G}_{F v, H y_{n}, R z_{n}}^{*}>\min \left\{G_{f v, h y_{n}, r z_{n}, \frac{3}{k}}^{*}\left[\widetilde{G}_{F v, h y_{n}, r z_{n}}^{*} \oplus \widetilde{G}_{f v, H y_{n}, r z_{n}}^{*} \oplus \widetilde{G}_{f v, h y_{n}, R z_{n}}^{*}\right]\right\} .
$$

It is easy to verify that

$$
\liminf _{n \rightarrow \infty}\left[\widetilde{G}_{F v, h y_{n}, r z_{n}}^{*} \oplus \widetilde{G}_{f v, H y_{n}, r z_{n}}^{*} \oplus \widetilde{G}_{f v, h y_{n}, R z_{n}}^{*}\right]\left(\frac{3}{k} t_{0}\right) \geq \widetilde{G}_{F v, u, u}^{*}\left(\frac{3}{k} t_{0}\right) .
$$


In fact, for any $\delta_{1}, \delta_{2} \in\left(0, \frac{3}{k} t_{0}\right)$, we have

$$
\begin{aligned}
& {\left[\widetilde{G}_{F v, h y_{n}, r z_{n}}^{*} \oplus \widetilde{G}_{f v, H y_{n}, r z_{n}}^{*} \oplus \widetilde{G}_{f v, h y_{n}, R z_{n}}^{*}\right]\left(\frac{3}{k} t_{0}\right)} \\
& \quad \geq \min \left\{\widetilde{G}_{F v, h y_{n}, r z_{n}}^{*}\left(\frac{3}{k} t_{0}-\delta_{1}-\delta_{2}\right), \widetilde{G}_{f v, H y_{n}, r z_{n}}^{*}\left(\delta_{1}\right), \widetilde{G}_{f v, h y_{n}, R z_{n}}^{*}\left(\delta_{2}\right)\right\} .
\end{aligned}
$$

Since $f v=u \in\left[\left(B=\lim _{n \rightarrow \infty} H y_{n}\right) \cap\left(C=\lim _{n \rightarrow \infty} R y_{n}\right)\right]$, by Lemma 1.3 and Theorem 2.2(1), we get

$$
\liminf _{n \rightarrow \infty}\left[\widetilde{G}_{F v, h y_{n}, r z_{n}}^{*} \oplus \widetilde{G}_{f v, H y_{n}, r z_{n}}^{*} \oplus \widetilde{G}_{f v, h y_{n}, R z_{n}}^{*}\right]\left(\frac{3}{k} t_{0}\right) \geq \widetilde{G}_{F v, u, u}^{*}\left(\frac{3}{k} t_{0}-\delta_{1}-\delta_{2}\right) .
$$

Letting $\delta_{1}, \delta_{2} \rightarrow 0$, by the left continuity of the distribution function, we obtain (3.5).

Noting that $t_{0}$ is the continuous point of $\widetilde{G}_{F v, B, C}^{*}(\cdot)$, by Lemma 1.3 , we have

$$
\lim _{n \rightarrow \infty} \widetilde{G}_{F v, H y_{n}, R z_{n}}^{*}\left(t_{0}\right)=\widetilde{G}_{F v, B, C}^{*}\left(t_{0}\right)
$$

Thus, letting $n \rightarrow \infty$ in (3.4) and using (3.5), we obtain

$$
\widetilde{G}_{F v, B, C}^{*}\left(t_{0}\right) \geq \min \left\{1, \widetilde{G}_{F v, u, u}^{*}\left(\frac{3}{k} t_{0}\right)\right\}=\widetilde{G}_{F v, u, u}^{*}\left(\frac{3}{k} t_{0}\right),
$$

that is,

$$
\widetilde{G}_{F v, B, C}^{*}\left(t_{0}\right) \geq \widetilde{G}_{F v, u, u}^{*}\left(\frac{3}{k} t_{0}\right)
$$

But since $f v \in B$, by Theorem 2.2(3) and (3.3), we obtain

$$
\widetilde{G}_{F v, u, u}^{*}\left(\frac{3}{k} t_{0}\right)>\widetilde{G}_{F v, B, C}^{*}\left(t_{0}\right)
$$

which is a contradiction. So, we get $f v \in F v$.

On the other hand, since $h(X)$ is $\mathscr{T}$-closed, there exists some $w \in X$, such that $u=h w$. We claim that $h w \in H w$. Suppose this is not true, that is, $h w \notin H w$. Noting that $u=h w \in C$, we have $C \neq H w$. Similarly, we know that there exists some $t_{1}>0$, such that

$$
\widetilde{G}_{F v, H w, C}^{*}\left(\frac{3}{k} t_{1}\right)>\widetilde{G}_{F v, H w, C}^{*}\left(t_{1}\right) .
$$

Similarly, without loss of generality, we can assume that $t_{1}$ is a continuous point of $\widetilde{G}_{F v, H w, C}^{*}(\cdot)$.

Noting that $\lim _{n \rightarrow \infty} r z_{n}=u \notin H w$ and $u \in C=\lim _{n \rightarrow \infty} R z_{n}$, there exists some $n_{1} \in \mathbb{Z}^{+}$, such that for all $n \geq n_{1}, R z_{n} \neq H w$.

From (3.1) we know that

$$
\widetilde{G}_{F v, H w, R z_{n}}^{*}\left(t_{1}\right)>\min \left\{G_{f v, h w, r z_{n}}^{*}\left(t_{1}\right),\left[\widetilde{G}_{F v, h w, r z_{n}}^{*} \oplus \widetilde{G}_{f v, H w, r z_{n}}^{*} \oplus \widetilde{G}_{f v, h w, R z_{n}}^{*}\right]\left(\frac{3}{k} t_{1}\right)\right\} .
$$


Similarly, we can verify that

$$
\liminf _{n \rightarrow \infty}\left[\widetilde{G}_{F v, H w, r z_{n}}^{*} \oplus \widetilde{G}_{f v, H w, r z_{n}}^{*} \oplus \widetilde{G}_{f v, h w, R z_{n}}^{*}\right]\left(\frac{3}{k} t_{1}\right) \geq \widetilde{G}_{u, H w, u}^{*}\left(\frac{3}{k} t_{1}\right) .
$$

Noting that $t_{1}$ is a continuous point of $\widetilde{G}_{F v, H w, C}^{*}(\cdot)$, by Lemma 1.3 , we have

$$
\lim _{n \rightarrow \infty} \widetilde{G}_{F v, H w, R z_{n}}^{*}\left(t_{1}\right)=\widetilde{G}_{F v, H w, C}^{*}\left(t_{1}\right) .
$$

Thus, letting $n \rightarrow \infty$ in (3.7) and using (3.8), we obtain

$$
\widetilde{G}_{F v, H w, C}^{*}\left(t_{1}\right) \geq \min \left\{1, \widetilde{G}_{u, H w, u}^{*}\left(\frac{3}{k} t_{1}\right)\right\}=\widetilde{G}_{u, H w, u}^{*}\left(\frac{3}{k} t_{1}\right) \geq \widetilde{G}_{F v, H w, C}^{*}\left(\frac{3}{k} t_{1}\right),
$$

which is a contradiction. So, we get $h w \in H w$.

Since $r(X)$ is $\mathscr{T}$-closed, there exists some $a \in X$, such that $u=r a$. We claim that $r a \in R a$. Suppose this is not true, that is, $r a \notin R a$. Noting that $u=r a \in A$, we have $A \neq R a$. Similarly, we know that there exists some $t_{2}>0$, such that

$$
\widetilde{G}_{A, H w, R a}^{*}\left(\frac{3}{k} t_{2}\right)>\widetilde{G}_{A, H w, R a}^{*}\left(t_{2}\right) .
$$

Similarly, without loss of generality, we can assume that $t_{2}$ is a continuous point of $\widetilde{G}_{A, H w, R a}^{*}(\cdot)$.

Noting that $\lim _{n \rightarrow \infty} f x_{n}=u \notin R a$ and $u \in A=\lim _{n \rightarrow \infty} F x_{n}$, there exists some $n_{2} \in \mathbb{Z}^{+}$, such that for all $n \geq n_{2}, F x_{n} \neq R a$.

From (3.1), we know that

$$
\widetilde{G}_{F x_{n}, H w, R a}^{*}\left(t_{2}\right)>\min \left\{G_{f x_{n}, h w, r a}^{*}\left(t_{2}\right),\left[\widetilde{G}_{F x_{n}, h w, r a}^{*} \oplus \widetilde{G}_{f x_{n}, H w, r a}^{*} \oplus \widetilde{G}_{f x_{n}, h w, R a}^{*}\right]\left(\frac{3}{k} t_{2}\right)\right\} .
$$

Similarly, it is easy to prove that $u=r a \in R a$. This implies that $v$ is a coincidence point of $(f, F), w$ is a coincidence point of $(h, H)$, and $a$ is a coincidence point of $(r, R)$.

Since $v \in C(f, F), w \in C(h, H)$, and $a \in C(r, R)$, we have $u=f v=f f v=f u \in F v, u=h w=$ $h h w=h u \in H w$, and $u=r a=r r a=r u \in R w$. Next, we prove that $F v=F u, H w=H u$, and $R a=R u$.

(1) First, we assert that $F v=H w$. In fact, suppose that $F v \neq H w$. Then, by (3.1), there exists some $t_{3}>0$, such that

$$
\widetilde{G}_{F v, H w, R a}^{*}\left(t_{3}\right)>\min \left\{G_{f v, h w, r a}^{*}\left(t_{3}\right),\left[\widetilde{G}_{F v, h w, r a}^{*} \oplus \widetilde{G}_{f v, H w, r a}^{*} \oplus \widetilde{G}_{f v, h w, R a}^{*}\right]\left(\frac{3}{k} t_{3}\right)\right\} .
$$

This implies that

$$
\widetilde{G}_{F v, H w, R a}^{*}\left(t_{3}\right)>1 \text {, }
$$

which is a contradiction, and thus we have $F v=H w$.

(2) Next, we assert that $F u=H w$. In fact, suppose that $F u \neq H w$. Then, by (3.1), there exists some $t_{4}>0$, such that

$$
\widetilde{G}_{F u, H w, R a}^{*}\left(t_{4}\right)>\min \left\{G_{f u, h w, r a}^{*}\left(t_{4}\right),\left[\widetilde{G}_{F u, h w, r a}^{*} \oplus \widetilde{G}_{f u, H w, r a}^{*} \oplus \widetilde{G}_{f u, h w, R a}^{*}\right]\left(\frac{3}{k} t_{4}\right)\right\} .
$$


This implies that

$$
\widetilde{G}_{F u, H w, R a}^{*}\left(t_{4}\right)>1 \text {, }
$$

which is a contradiction, and thus we have $F u=H w$. Combining these two facts yields $F v=F u$. Similarly, we can prove that $H w=R a=H u$ and $R a=F v=R u$. Thus, we have $u=f u \in F u, u=h u \in H u$, and $u=r u \in R u$, that is, $u$ is the common fixed point of $f, h, r$, $F, H$, and $R$. This completes the proof.

Setting $f=h=r$ and $F=H=R$, we obtain the following result.

Theorem 3.2 Let $\left(X, G^{*}, \Delta\right)$ be a Menger PGM-space with a continuous $t$-norm on $[0,1] \times$ $[0,1]$ and $\left(\Omega^{*}, \widetilde{G}^{*}, \Delta\right)$ be the induced Menger PGM-space. Suppose that $f: X \rightarrow X$ and $F: X \rightarrow \Omega^{*}$ are mappings satisfying the following conditions:

(1) $(f, F)$ satisfies the property $(E . A)$;

(2) $f(X)$ is a $\mathscr{T}$-closed subset of $X$;

(3) for any $x, y, z \in X$ with $F x, F y$, and $F z$ not all equal and some $1 \leq k \leq 3$,

$$
\widetilde{G}_{F x, F y, F z}^{*}(t)>\min \left\{G_{f x, f y, f z}^{*}, \frac{3}{k}\left[\widetilde{G}_{F x, f y, f z}^{*} \oplus \widetilde{G}_{f x, F y, f z}^{*} \oplus \widetilde{G}_{f x, f y, F z}^{*}\right]\right\}
$$

where $\frac{3}{k}\left[\widetilde{G}_{F x, f y, f z}^{*} \oplus \widetilde{G}_{f x, F y, f z}^{*} \oplus \widetilde{G}_{f x, f y, F z}^{*}\right](t)$ means $\left[\widetilde{G}_{F x, f y, f z}^{*} \oplus \widetilde{G}_{f x, F y, f z}^{*} \oplus \widetilde{G}_{f x, f y, F z}^{*}\right]\left(\frac{3}{k} t\right)$.

Then $f$ and $F$ have a coincidence point. Moreover, if ffv $=f v$ for $v \in C(f, F)$, then $f$ and $F$ have a common fixed point in $X$.

\section{An example}

In this section, we will provide an example to show the validity of Theorem 3.1.

Example 4.1 Let $X=(-2,2)$ and define

$$
\begin{aligned}
& G_{x, y, z}^{*}(t)=\frac{t}{t+\max \{|x-y|,|y-z|,|z-x|\}}, \\
& \widetilde{G}_{A, B, C}^{*}(t)=\frac{t}{t+\max \{\delta(A, B), \delta(B, C), \delta(A, C)\}}
\end{aligned}
$$

for all $x, y, z \in X, A, B, C \in \Omega^{*}$, and $t \geq 0$. Then, by Example 2.1, $\left(X, G^{*}, \Delta_{m}\right)$ and $\left(\Omega^{*}, \widetilde{G}^{*}, \Delta_{m}\right)$ are $P G M$-spaces. Define $f, h, r: X \rightarrow X$ and $F, H, R: X \rightarrow \Omega^{*}$ as follows:

$$
\begin{aligned}
& f x=\left\{\begin{array}{ll}
\frac{5}{6}, & x \in(-2,-1) \cup(1,2) ; \\
\frac{1}{3} x, & x \in[-1,1],
\end{array} \quad F x= \begin{cases}{\left[0, \frac{2}{3}\right],} & x \in(-2,-1) \cup(1,2) \\
{\left[\frac{1}{3} x, 0\right],} & x \in[-1,0] \\
{\left[0, \frac{1}{3} x\right],} & x \in[0,1]\end{cases} \right. \\
& h x=\left\{\begin{array}{ll}
\frac{4}{5}, & x \in(-2,-1) \cup(1,2) ; \\
\frac{1}{3} x, & x \in[-1,1],
\end{array} \quad H x= \begin{cases}{\left[0, \frac{1}{2}\right],} & x \in(-2,-1) \cup(1,2) ; \\
{\left[0,-\frac{1}{4} x\right],} & x \in[-1,0] ; \\
{\left[-\frac{1}{4} x, 0\right],} & x \in[0,1],\end{cases} \right. \\
& r x=\left\{\begin{array}{ll}
\frac{7}{8}, & x \in(-2,-1) \cup(1,2) ; \\
\frac{1}{3} x, & x \in[-1,1],
\end{array} \quad R x= \begin{cases}{\left[0, \frac{3}{4}\right],} & x \in(-2,-1) \cup(1,2) ; \\
{\left[\frac{2}{3} x, 0\right],} & x \in[-1,0] ; \\
{\left[0, \frac{2}{3} x\right],} & x \in[0,1] .\end{cases} \right.
\end{aligned}
$$


Consider the sequences $\left\{x_{n}=\frac{1}{n+1}\right\}$ and $\left\{y_{n}=-\frac{1}{n+1}\right\}$ in $X$. Then

$$
\lim _{n \rightarrow \infty} f x_{n}=\lim _{n \rightarrow \infty} h x_{n}=\lim _{n \rightarrow \infty} r x_{n}=0 \in \lim _{n \rightarrow \infty} F x_{n} \cap \lim _{n \rightarrow \infty} H x_{n} \cap \lim _{n \rightarrow \infty} R x_{n}
$$

which shows that $(f, F),(h, H)$, and $(r, R)$ satisfy the common property $(E . A)$. Also $f(X)$, $h(X)$, and $r(X)$ are $\mathscr{T}$-closed subsets of $X$. By a routine calculation, one can verify that (3.1) holds for all $x, y, z \in X, t>0$, and some $1 \leq k<3$.

In fact, if $x, y, z \in(-2,-1) \cup(1,2)$, for any $t>0$,

$$
\begin{aligned}
& \widetilde{G}_{F x, H y, R z}^{*}(t)=\frac{t}{t+\max \left\{\delta\left(\left[0, \frac{2}{3}\right],\left[0, \frac{1}{2}\right]\right), \delta\left(\left[0, \frac{3}{4}\right],\left[0, \frac{1}{2}\right]\right), \delta\left(\left[0, \frac{2}{3}\right],\left[0, \frac{3}{4}\right]\right)\right\}}=\frac{t}{t+0}=1, \\
& \widetilde{G}_{f x, h y, r z}^{*}(t)=\frac{t}{t+\max \left\{\left(\frac{5}{6}-\frac{4}{5}\right),\left(\frac{7}{8}-\frac{4}{5}\right),\left(\frac{7}{8}-\frac{5}{6}\right)\right\}}=\frac{t}{t+\frac{3}{40}}<1 .
\end{aligned}
$$

So, we have

$$
\widetilde{G}_{F x, H y, R z}^{*}(t)>\widetilde{G}_{f x, h y, r z}^{*}(t) \geq \min \left\{G_{f x, h y, r z}^{*}(t), \frac{3}{k}\left[\widetilde{G}_{F x, h y, r z}^{*} \oplus \widetilde{G}_{f x, H y, r z}^{*} \oplus \widetilde{G}_{f x, h y, R z}^{*}\right](t)\right\} .
$$

Similarly, if $x, y, z \in[-1,0]$, or $x, y, z \in[0,1]$, we also have

$$
\widetilde{G}_{F x, H y, R z}^{*}(t)=1>\widetilde{G}_{f x, h y, r z}^{*}(t) \geq \min \left\{G_{f x, h y, r z}^{*}(t),{ }_{\frac{3}{k}}\left[\widetilde{G}_{F x, h y, r z}^{*} \oplus \widetilde{G}_{f x, H y, r z}^{*} \oplus \widetilde{G}_{f x, h y, R z}^{*}\right](t)\right\} .
$$

If $x, y \in(-2,-1) \cup(1,2), z \in[0,1]$, we have

$$
\begin{aligned}
& \widetilde{G}_{F x, H y, R z}^{*}(t)=\frac{t}{t+\max \left\{\delta\left(\left[0, \frac{2}{3}\right],\left[0, \frac{1}{2}\right]\right), \delta\left(\left[\frac{1}{4} x, 0\right],\left[0, \frac{1}{2}\right]\right), \delta\left(\left[0, \frac{2}{3}\right],\left[\frac{1}{4} x, 0\right]\right)\right\}}=\frac{t}{t+0}=1, \\
& \widetilde{G}_{f x, h y, r z}^{*}(t)=\frac{t}{t+\max \left\{\left(\frac{5}{6}-\frac{4}{5}\right),\left|\frac{1}{3} z-\frac{4}{5}\right|,\left|\frac{1}{3} z-\frac{5}{6}\right|\right\}} \leq \frac{t}{t+\frac{1}{30}}<1 .
\end{aligned}
$$

So, we have

$$
\widetilde{G}_{F x, H y, R z}^{*}(t)>\widetilde{G}_{f x, h y, r z}^{*}(t) \geq \min \left\{G_{f x, h y, r z}^{*}(t), \frac{3}{k}\left[\widetilde{G}_{F x, h y, r z}^{*} \oplus \widetilde{G}_{f x, H y, r z}^{*} \oplus \widetilde{G}_{f x, h y, R z}^{*}\right](t)\right\} .
$$

Similarly, it is easy to verify (3.1) for the other cases. Thus, all the conditions of Theorem 3.1 are satisfied and 0 is the unique coincidence point of $(f, F),(h, H)$, and $(r, R)$. Furthermore, noting that $f f 0=f 0, h h 0=h 0$, and $r r 0=r 0,0$ remains the common fixed point of $(f, F),(h, H)$, and $(r, R)$.

\section{Competing interests}

The authors declare that they have no competing interests.

Authors' contributions

All authors contributed equally. All authors read and approved the final manuscript.

\section{Acknowledgements}

The authors would like to thank the editor and the referees for their constructive comments and suggestions. The research was supported by the National Natural Science Foundation of China $(11361042,11326099,11461045,11071108)$ and the Provincial Natural Science Foundation of Jiangxi, China (20132BAB201001, 20142BAB211016, 2010GZS0147). 


\section{References}

1. Menger, K: Statistical metrics. Proc. Natl. Acad. Sci. USA 28, 535-537 (1942)

2. Schweizer, B, Sklar, A: Statistical metric spaces. Pac. J. Math. 10, 313-334 (1960)

3. Zhu, CX: Several nonlinear operator problems in the Menger PN space. Nonlinear Anal. 65(7), 1281-1284 (2006)

4. Zhu, CX: Research on some problems for nonlinear operators. Nonlinear Anal. 71(10), 4568-4571 (2009)

5. Egbert, RJ: Products and quotients of probabilistic metric spaces. Pac. J. Math. 24(3), 437-455 (1968)

6. Mustafa, Z, Sims, B: A new approach to generalized metric spaces. J. Nonlinear Convex Anal. 7(2), 289-297 (2006)

7. Gajić, L, Stojaković, M: On mappings with $\varphi$-contractive iterate at a point on generalized metric spaces. Fixed Point Theory Appl. 2014, 46 (2014)

8. Gu, F, Shatanawi, W: Common fixed point for generalized weakly G-contraction mappings satisfying common property in G-metric spaces. Fixed Point Theory Appl. 2013, 309 (2013)

9. Bilgili, N, Karapinar, E: Cyclic contractions via auxiliary functions on G-metric spaces. Fixed Point Theory Appl. 2013, 49 (2013)

10. Gu, F, Yang, Z: Some new common fixed point results for three pairs of mappings in generalized metric spaces. Fixed Point Theory Appl. 2013, 174 (2013)

11. Shatanawi, W, Pitea, A: Fixed and coupled fixed point theorems of omega-distance for nonlinear contraction. Fixed Point Theory Appl. 2013, 275 (2013)

12. Karapinar, E, Agarwal, RP: Further fixed point results on G-metric spaces. Fixed Point Theory Appl. 2013, 154 (2013)

13. Kaewcharoen, A, Kaewkhao, A: Common fixed points for single-valued and multi-valued mappings in G-metric spaces. Int. J. Math. Anal. 5(36), 1775-1790 (2011)

14. Zhou, C, Wang, S, Ćirić, L, Alsulami, SM: Generalized probabilistic metric spaces and fixed point theorems. Fixed Point Theory Appl. 2014, 91 (2014)

15. Zhu, $C X, X u, W Q, W u, Z Q:$ Some fixed point theorems in generalized probabilistic metric spaces. Abstr. Appl. Anal. 2014, $103764(2014)$

16. Chang, SS, Cho, YJ, Kang, SM: Nonlinear Operator Theory in Probabilistic Metric Spaces. Nova Science Publishers, New York (2001)

17. Jungck, G: Common fixed points for noncontinuous nonself mappings on nonnumeric spaces. Far East J. Math. Sci. 4(2), 199-221 (1996)

18. Fang, JX, Gang, Y: Common fixed point theorems under strict contractive conditions in Menger spaces. Nonlinear Anal. 70(1), 184-193 (2009)

19. Jungck, G, Rhoades, BE: Fixed points for set valued functions without continuity. Indian J. Pure Appl. Math. 29(3), 227-238 (1998)

20. $\mathrm{Wu}, \mathrm{ZQ}, \mathrm{Zhu}, \mathrm{CX}, \mathrm{Li}, \mathrm{J}$ : Common fixed point theorems for two hybrid pairs of mappings satisfying the common property (E.A) in Menger PM-space. Fixed Point Theory Appl. 2013, 25 (2013)

\section{Submit your manuscript to a SpringerOpen ${ }^{\circ}$ journal and benefit from:}

- Convenient online submission

- Rigorous peer review

- Immediate publication on acceptance

- Open access: articles freely available online

- High visibility within the field

- Retaining the copyright to your article 